\title{
UN CAS DE LADRERIE CAPRINE
}

\author{
Par le Dr L. FAURE \\ Vétérinaire-Capitaiue, Chef de service de la place de Marseille
}

Dans les ouvrages classiques de parasitologie vétérinaire, les caprins ne figurent point au nombre des mammifères susceptibles de contracter spontanément la Cysticercose. L.-G. Neumann, dans son traité des maladies parasitaires, signale simplement la possibilité d'infecter expérimentalement la chèvre : "Zenker, dit-il, a déterminé la ladrerie chez trois chèvres auxquelles il avait fait prendre des anneaux de Tænia saginata; Heller a eu aussi des résultats positifs sur deux chèvres et un mouton. »Il mentionne par contre les tentatives infructueuses de Zürn pour obtenir la cysticercose expérimentale chez la chèvre.

L'observation suivante d'un cas de ladrerie spontanée, chez une chèvre indigène adulte du Sud-Tunisien, mérite d'être rapportée en raison de son extrême rareté.

Distribution, abondance et caractères généraux des lésions. L'examen du cour attire d'abord l'attention. Une quarantaine de nodosités, plus ou moins développées, sont réparties à la surface de l'organe. A côté de tubercules opaques, jaunâtres, minuscules (variant de la grosseur d'une tête d'épingle à celle d'un grain de mil), à peine enchâssés dans les fibres myocardiques, quelques autres atteignent le volume d'une lentille et même d'un petit pois.

En sectionnant diamétralement l'organe on note l'existence de kystes similaires, en nombre plus restreint toutefois, dans l'épaisseur du myocarde et à l'intérieur des cavités cardiaques, en saillie sous l'endocarde. La coupe a intéressé plusieurs nodules: le contenu des petits kystes opaques, dégénérés, a l'aspect de mastic compact et ne fournit macroscopiquement aucune donnée caractéristique sur la nature intime de la lésion; des logettes plus spacieuses, affaissées par l'incision, s'écoule une minime quantité de liquide clair ; tapissant la cavité, une membrane non adhérente, facilement énucléable, indique l'origine parasitaire probable de ces vésicules, à divers stades d'évolution.

L’importance des lésions cardiaques fait rechercher leur répartition dans les autres tissus de l'organisme. Tous les autres viscè-

Annales de Parasitologie, $\mathrm{T} . \mathrm{XIV}, \mathrm{N}^{0} 5 .-1^{\mathrm{er}}$ septembre 1936. 
res paraissent indemnes. Rien de particulier non plus à l'inspection des fentes vertébrale et sternale, ni sur les coupes musculaires obtenues par décapitation. Une vésicule ladrique isolée est logée dans la partie charnue du diaphragme, sous la séreuse péritonéale. A la faveur d'incisions complémentaires sont découverts cinq autres grains lenticulaires, jaunâtres, opaques, de consistance ferme: deux siègent dans les anconés, un dans le pectoral superficiel, un dans le corps lingual et le dernier dans le masséter. Cachés au sein des muscles, ces « grains», pour un observateur non prévenu, passeraient inaperçus. Bien qu'ils ne soient pas infiltrés de sels calcaires, leur dégénérescence est manifeste et ils sont certaine-

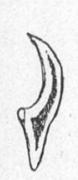

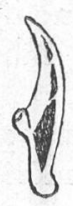

FIG. 1. - Petit crochet. Grand crochet

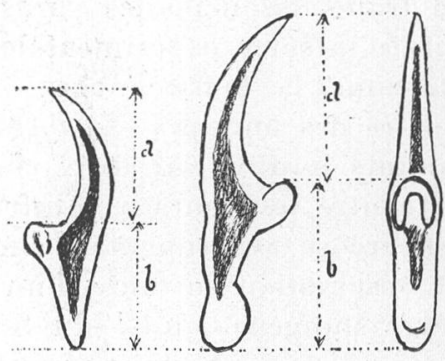

FIG. 2. - Les mêmes crochets à plus fort grossissement

ment inaptes à provoquer une téniose par ingestion de la viande.

Comme la saisie ne s'impose pas, il nous est difficile de morceler davantage la carcasse, aussi tous les parasites ne sont-ils pas dénombrés, mais nos investigations suffisent à conclure à une invasion discrète.

Morphologie et identification du parasite. - Nul n'ignore les interprétations variables auxquelles ont donné lieu les rares observations de ladrerie ovine. Plusieurs parasites, d'après le professeur A. Henry (Bulletin de la Société Centrale de médecine vétérinaire, 30 novembre 1913 , p. 410), peuvent provoquer cette cysticercose, notamment le C. cellulosæ; et ainsi se trouvent justifiées les prescriptions sanitaires appliquées aux autres ladreries.

Les recherches relatives à la nature du parasite en cause n'ont pas un intérêt purement théorique, car au point de vue de l'hygiène publique, l'inspecteur des viandes a besoin d'être éclairé.

Dans le cas qui nous occupe, les examens microscopiques ont 
porté sur les vésicules extraites des alvéoles myocardiques ayant atteint un grand développement. Les membranes vésiculaires présentent un prolongement blanchâtre pourvu d'un renflement terminal, lequel correspond au scolex. Le segment parasitaire qui fait suite au cou est littéralement bourré de corpuscules ovoïdes simulant des oeufs minuscules. Quatre ventouses ovalaires occupent la partie renflée de la tête, tandis que deux couronnes de cro-
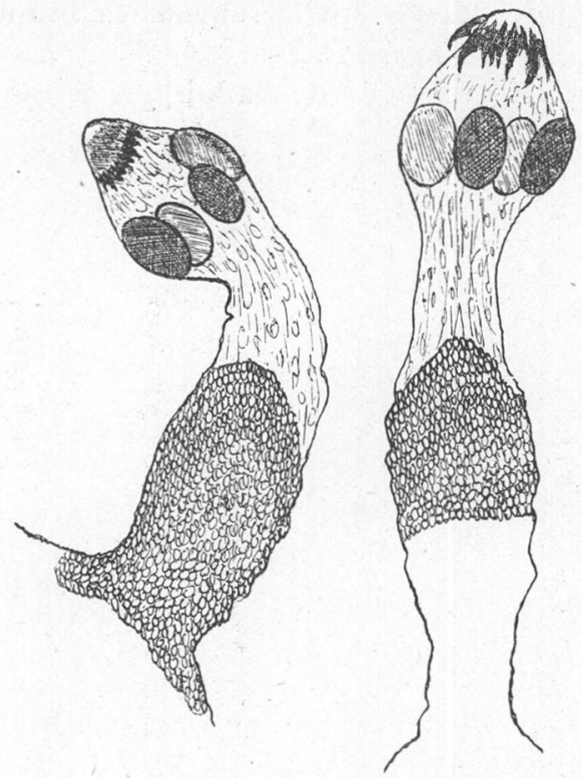

Fig, 3. - Scolex armés à 4 ventouses.

chets sont implantées dans le rostre. Ces parasites sont donc des formes vivantes de cysticerques armés.

Pour permettre une comparaison avec les types classiques étudiés et figurés dans la revue professionnelle précitée, à la description des caractères morphologiques sont joints des dessins de scolex, puis des crochets à divers grossissements. En l'absence de micromètre objectif nous n'avons pu effectuer des mensurations précises (1). Séparés par une garde saillante, le manche et la lame ont une faible différence de longueur à l'avantage de cette dernière.

(1) La longueur des grands crochets est de $142 \mu, 5$ à $150 \mu$, celle des petits crochets de 96 à $116 \mu$ (R.-Ph. D.). 
A partir du milieu du manche, la lame des petits crochets se redresse dorsalement, se déjette nettement en arrière ; la pulpe centrale s'étend assez loin vers chacune des extrémités.

Après examen et figuration à plat, par de légers déplacements imposés à la lamelle, un grand crochet isolé a pu être disposé de profil ; dans cette position, la lame dessine un cône effilé à peu près régulier, tandis que deux droites sensiblement parallèles profilent le manche. A leur point d'union, marqué par un très léger renflement, la garde dessine un $\mathrm{U}$ renversé à branches trapues et resserrées.

A notre avis, c'est avec ceux de Ballon que le cysticerque caprin offre le plus d'analogie. 